\title{
Physicochemical Properties of Rice Flour Suspension Treated by Ultrahigh Hydrostatic Pressure
}

\author{
Ji Won Park, ${ }^{1}$ Jeong Hyun Seo, ${ }^{1}$ Chae Young Hong, ${ }^{1}$ Min Young Kim, ${ }^{2}$ Yoon Jeong Lee, ${ }^{3}$ \\ A.-Reum Chun, ${ }^{2}$ Youn Ri Lee, ${ }^{4}$ Junsoo Lee $\mathbb{D}^{1},{ }^{1}$ and Heon Sang Jeong $\mathbb{D D}^{1}$ \\ ${ }^{1}$ Department of Food Science and Biotechnology, Chungbuk National University, Cheongju, Chungbuk 28644, Republic of Korea \\ ${ }^{2}$ Department of Central Area Crop Science, National Institute of Crop Science, Rural Development Administration, Suwon, \\ Gyeonggi 16429, Republic of Korea \\ ${ }^{3}$ Department of Herbal Crop Research, National Institute of Horticultural \& Herbal Science, Eumsung, \\ Chungbuk 27709, Republic of Korea \\ ${ }^{4}$ Department of Food and Nutrition, Daejeon Health Sciences College, Daejeon 34504, Republic of Korea
}

Correspondence should be addressed to Heon Sang Jeong; hsjeong@chungbuk.ac.kr

Received 16 June 2020; Revised 25 January 2021; Accepted 15 February 2021; Published 8 March 2021

Academic Editor: Flora V. Romeo

Copyright (c) 2021 Ji Won Park et al. This is an open access article distributed under the Creative Commons Attribution License, which permits unrestricted use, distribution, and reproduction in any medium, provided the original work is properly cited.

This study investigated the physicochemical properties of rice flour suspensions under ultrahigh hydrostatic pressure (UHP) treatment. Rice flour suspensions were subjected to 200, 400, and $600 \mathrm{MPa}$ of pressure for $10 \mathrm{~min}$, and heat treatment was used as a control. Proximate characteristics of different rice cultivar were analyzed to amylose, damage starch content, and particle size. Changes in physicochemical properties of rice flour suspensions according to UHP treatment were analyzed to microscopic structure, iodine reaction, $\alpha$-amylase hydrolysis rate, and resistant starch content. Microscopic structural analyses showed that the structures of the rice flours were altered under both heat and $600 \mathrm{MPa}$ treatment conditions. Water absorption rates were highest under heat treatment (467.53-554.85\%), followed by $600 \mathrm{MPa}$ treatment (269.55-334.57\%). Iodine reaction values increased with increasing applied pressure. $\alpha$-Amylase hydrolysis rates and resistant starch contents were highest under heat treatment and increased with increasing applied pressure. Based on these results, $600 \mathrm{MPa}$ treatment of rice flour suspensions was shown to be comparable to heat treatment; as a result, the development of the new rice processing method with different physicochemical properties is expected from rice cultivars treated under UHP processing methods.

\section{Introduction}

Ultrahigh hydrostatic pressure (UHP) refers to pressure above the maximum pressure $(110 \mathrm{MPa})$ found in nature. UHP treatment involves sealing a liquid or solid food into a flexible container and applying high pressure via water as a pressure transfer medium. Pressure applies equally to all parts of a sample, regardless of size, shape, or time. And pressurization leads to extinction of microorganisms, changes in enzymatic reaction rates, reversible and irreversible inactivation of enzymes, and gelation of proteins and starches [1].

Nonthermal processes are evolving as potential alternatives to thermal and chemical operations in food- processing methods. UHP is a typical nonthermal process for food preservation. Pressure is a quantifiable energy, like temperature is to heat; therefore, pressure can be used as a thermodynamic factor of changes in the state of a material. However, unlike heat treatment, covalent bond formation and breakage does not occur under high pressure treatment, which affects only noncovalent bonds, retaining nutrients at room temperature [2]. UHP technologies have been developed extensively for food processing and can address the needs of modern consumers who prefer minimally processed foods [3].

Rice (Oryza sativa L.) crop serves as a staple food of Korea, Asia, Africa, and Latin America and is an important grain consumed as more than half of the calories per day in 
Asia. However, rice consumption has steadily decreased due to the emergence of alternative and various instant processed foods. Recently, rice varieties with improved functionalities and processabilities have been developed to promote rice consumption. To this end, it is therefore necessary to develop and materialize new properties and varieties of rice. Processed type rice, distinct from normal type rice, is developed for the industries of rice cakes, noodles, baking, and brewing [4]. Therefore, differences in starch structure and gelatinization properties between these two types of rice exist. Most rice is processed by gelatinization through heat treatment, but recently, methods of gelatinization through nonthermal treatments have been studied. Therefore, it is necessary to confirm the properties of rice cultivars undergoing nonthermal treatment gelatinization. It is reported that starch gelatinization occurs at applied pressures of more than $400 \mathrm{MPa}$ [5].

Therefore, the aims of this study were to investigate the physicochemical properties of rice flour suspensions under UHP treatment and develop a correlation between the gelatinization and physicochemical properties of rice cultivars under this method.

\section{Materials and Methods}

2.1. Materials and Suspension Preparation. The rice used in this study was normal type cultivar Samkwang and processing type cultivars Shingil and Hangaru. The rice cultivars were provided by the Rural Development Administration (Jeonju, Jeollabuk-do, Republic of Korea). Samkwang is hard rice, with a polygonal starch structure. Shingil and Hangaru are soft rice, with round starch structures, like those of wheat starch. Corn starch (Tureban Co., Ltd., Goyang, Republic of Korea) was purchased from the market and used as a control. The three rice cultivars were dry milled using Air Mill (MCM-3, Nara machinery Co., LTD., Tokyo, Japan). The suspensions were composed of distilled water at concentrations of $15 \%$ [6].

2.2. Measurement of Amylose Content. Amylose content was evaluated using the method described by Juliano [7]. The rice flours (100 mg) were placed in volumetric flasks, and $1 \mathrm{~mL}$ of $95 \%$ ethanol and $9 \mathrm{~mL}$ of $1 \mathrm{M} \mathrm{NaOH}$ were added. The suspensions were heated in a boiling water bath for $10 \mathrm{~min}$, followed by cooling to room temperature. The flasks were then filled to the $100 \mathrm{~mL}$ mark with distilled water. A $5 \mathrm{~mL}$ aliquot of each diluted sample was collected. Then, $1 \mathrm{~mL}$ of $1 \mathrm{~N}$ acetic acid and $2 \mathrm{~mL}$ of $2 \%$ iodine solution $\left(0.2 \% \mathrm{w} / \mathrm{v} \mathrm{I}^{2}\right.$ in $2 \% \mathrm{KI}$ ) were added, and the solutions were adjusted to $100 \mathrm{~mL}$ with distilled water. The samples were evaluated using a spectrophotometer at a wavelength of $620 \mathrm{~nm}$. Different concentrations of amylose, free of amylopectin (Sigma-Aldrich, St. Louis, MO), were used to create a calibration curve.

2.3. Measurement of Damage Starch and Particle Size. Damaged starch content analyses were performed according to the AACC method [8] using a Megazyme kit (Megazyme,
Wicklow, Ireland). To $100 \mathrm{mg}$ of each rice flour sample, $50 \mathrm{U} / \mathrm{mL}$ of $\alpha$-amylase (Megazyme, Wicklow, Ireland) was added. The mixtures were then reacted at $40^{\circ} \mathrm{C}$ for $10 \mathrm{~min}$, followed by addition of $0.2 \%$ sulfuric acid. After centrifugation, amyloglucosidase (Megazyme, Wicklow, Ireland) was added to the supernatants to calculate the amounts of damaged starch present based on glucose contents generated after reaction at $40^{\circ} \mathrm{C}$ for $10 \mathrm{~min}$.

The particle sizes of the rice flours were measured using a Mastersizer 2000 (Malvern Instruments, Worcestershire, $\mathrm{UK})$. The rice flours were measured at a $0.03 \%$ concentration using ethanol as a dispersion solvent and expressed as an average particle size $(\mu \mathrm{m})$. Also, particle RI was measured at 1.52 and dispersant RI at 1.36 conditions.

2.4. Ultrahigh Hydrostatic Pressure Treatment. UHP treatment was performed using an ultrahigh pressure instrument (nonstirred autoclave system; Ilshin Autoclave Inc., Daejeon, Republic of Korea). The suspensions were packed without air in polyethylene film (New Pack, Seoul, Republic of Korea) and treated under pressures of 200, 400, and $600 \mathrm{MPa}$ and $25^{\circ} \mathrm{C}$ for $10 \mathrm{~min}$. Heat treatment at $90^{\circ} \mathrm{C}$ for $30 \mathrm{~min}$ was used as a control.

2.5. Measurement of Microscopic Structure. Microscopic structural analyses were performed on the UHP treatment suspensions. The samples were dried using a freeze dryer, then mounted on a scanning electron microscope (SEM) stub with double-sided adhesive tape, and coated with gold. Scanning electron micrographs were obtained using an ULTRA PLUS instrument (Carl Zeiss AG, Oberkochen, Germany).

2.6. Measurement of Moisture Absorption. Moisture absorption was measured using a modified method by Medcalf and Gilles [9]. The ultrahigh pressure and heat treated samples $(1 \mathrm{~g})$ with distilled water $(40 \mathrm{~mL})$ were placed in $50 \mathrm{~mL}$ centrifuge tubes and centrifuged at 3,500 rpm for $30 \mathrm{~min}$. The precipitates, obtained by centrifugation, were weighed to calculate moisture absorption as a ratio of sample weight prior to immersion.

2.7. Measurement of Iodine Reaction. Iodine reaction was measured using a modified method by Williams et al. [10]. To a $400 \mathrm{mg}$ aliquot of each suspension, $0.5 \mathrm{~N}$ of $\mathrm{KOH}$ $(10 \mathrm{~mL})$ was added and decentralized. Then, distilled water was added to a final volume of $100 \mathrm{~mL}$. To $10 \mathrm{~mL}$ aliquots of each diluted solution, $5 \mathrm{~mL}$ of $0.1 \mathrm{~N} \mathrm{HCl}$ and $0.5 \mathrm{~mL}$ of iodine solution $\left(0.2 \mathrm{~g} \mathrm{I}^{2}+2.0 \mathrm{~g} \mathrm{KI} / 100 \mathrm{~mL}\right.$ distilled water) were added and mixed. Distilled water was then added to a final volume of $50 \mathrm{~mL}$. After standing at room temperature for $20 \mathrm{~min}$, the absorbance of each solution was measured at $625 \mathrm{~nm}$.

2.8. Measurement of $\alpha$-Amylase Hydrolysis Rate. $\alpha$-Amylase hydrolysis rates were assessed using a modified method by Xue et al. [11]. Approximately, $50 \mathrm{mg}$ of each 
sample was diluted in $2 \mathrm{~mL}$ of $0.2 \mathrm{M}$ phosphate buffer $(\mathrm{pH}$ 6.9). Then, $0.5 \mathrm{~mL}$ of amylase buffer, prepared by dissolving $60 \mathrm{mg}$ of pancreatic $\alpha$-amylase (30 U/mg; Sigma-Aldrich, St. Louis, MO) in $50 \mathrm{~mL}$ of $0.2 \mathrm{M}$ phosphate buffer ( $\mathrm{pH}$ 6.9), was added. The samples were incubated at $37^{\circ} \mathrm{C}$ for $2 \mathrm{~h}$. Reducing sugars were estimated with 3,5-dinitrosalicylic acid reagent (Sigma-Aldrich, St. Louis, MO). After incubation, $4 \mathrm{~mL}$ of 3,5-dinitrosalicylic acid reagent was added to each sample. The samples were then heated at $100^{\circ} \mathrm{C}$ for $5 \mathrm{~min}$ and cooled to room temperature. To $300 \mu \mathrm{L}$ of each sample, $900 \mu \mathrm{L}$ of distilled water was added and mixed. Then, the samples were analyzed using a spectrophotometer at a wavelength of $550 \mathrm{~nm}$. Different concentrations of maltose were used to generate a calibration curve, and $\alpha$-amylase hydrolysis was expressed in terms of $\mathrm{mg}$ of maltose released/g of the sample.

2.9. Measurement of Resistant Starch Content. Resistant starch content analyses were performed using a Megazyme Resistant Starch Assay Kit (Megazyme, Wicklow, Ireland). Four milliliters of mixed $\alpha$-amylase and amyloglucosidase (Megazyme, Wicklow, Ireland) solution was added to $0.1 \mathrm{~g}$ of each sample, and the mixtures were left in a water bath (KMC-12055W1; Vision Scientific, Daejeon, Republic of Korea) at $200 \mathrm{rpm}$ and $37^{\circ} \mathrm{C}$ for $16 \mathrm{~h}$. Thereafter, $4 \mathrm{~mL}$ of $99 \%$ ethanol (Daejung Chemicals \& Metals Co., Ltd., Gyeonggi, Republic of Korea) was added to stop the enzymatic reaction. After centrifugation (3,000 rpm, $10 \mathrm{~min}), 2 \mathrm{~mL}$ of $2 \mathrm{M}$ $\mathrm{KOH}$ was added to the precipitates, which were then stirred for $20 \mathrm{~min}$ in an ice water bath. Neutralization was performed by adding 1.2 M sodium acetate buffer ( $\mathrm{pH} 3.8$; Sigma-Aldrich, St. Louis, MO) and $0.1 \mathrm{~mL}$ of amyloglucosidase to the samples, followed by heating in a water bath at $50^{\circ} \mathrm{C}$ for $30 \mathrm{~min}$. After centrifugation $(3,000 \mathrm{rpm}, 10 \mathrm{~min})$, $3 \mathrm{~mL}$ of glucose oxidase/peroxidase reagent (Megazyme, Wicklow, Ireland) was added to $0.1 \mathrm{~mL}$ of each supernatant and reacted for $20 \mathrm{~min}$ in a $50^{\circ} \mathrm{C}$ water bath. Absorbances were measured at $510 \mathrm{~nm}$ to determine glucose contents which were converted to resistant starch contents.

2.10. Statistical Analyses. All analyses were performed in triplicate and expressed as mean \pm SD. For statistical analyses, SPSS statistical software (Statistical Package for Social Science, Ver. 12.0; SPSS Inc., Chicago, IL) was used to calculate the mean and standard deviation of each treatment group, and Duncan's multiple range test was used to determine significance.

\section{Results and Discussion}

3.1. Amylose Content, Damaged Starch Content, and Particle Size of the Raw Rice Flours. The amylose contents of the rice flours depending on cultivars are shown in Table 1. The amylose content of corn starch was the highest, $27.80 \%$, while the Shingil cultivar had a significantly higher content, $22.98 \%$, than the other rice cultivars. These results are similar to those reported by Guo et al. [12], in which the amylose contents of rice and corn starch were approximately 20 and $28 \%$, respectively. According to the Rural Development
TABLe 1: Proximate compositions, amylose content, damaged starch content, and particle size of different rice flours.

\begin{tabular}{lccc}
\hline $\begin{array}{l}\text { Rice } \\
\text { cultivars }\end{array}$ & Amylose (\%) & $\begin{array}{c}\text { Damaged starch } \\
(\%)\end{array}$ & Particle size $(\mu \mathrm{m})$ \\
\hline Samkwang & $18.95 \pm 0.15^{1) \mathrm{c}}$ & $5.86 \pm 0.42^{\mathrm{a}}$ & $56.50 \pm 3.30^{\mathrm{a}}$ \\
Shingil & $22.98 \pm 0.80^{\mathrm{b}}$ & $4.29 \pm 0.33^{\mathrm{b}}$ & $46.10 \pm 3.20^{\mathrm{b}}$ \\
Hangaru & $19.71 \pm 0.68^{\mathrm{c}}$ & $3.79 \pm 0.31^{\mathrm{b}}$ & $52.90 \pm 3.70^{\mathrm{a}}$ \\
Corn starch & $27.80 \pm 0.59^{\mathrm{a}}$ & $0.49 \pm 0.08^{\mathrm{c}}$ & $39.25 \pm 3.32^{\mathrm{c}}$ \\
\hline
\end{tabular}

${ }^{1)}$ Values are mean \pm SD. $n=3 .{ }^{\mathrm{a}-c}$ Different small letters in the column indicate a significant difference by Duncan's range test $(p<0.05)$ according to the sample.

Administration, the amylose contents of Samkwang, Shingil, and Hangaru cultivars are 18.3, 23.4, and 19.2\%, respectively, which are similar to the values obtained in this experiment. Damaged starch content was significantly high in the Samkwang cultivar, 5.86\%, while corn starch had the lowest content, $0.49 \%$. Damaged starch is a small particle that breaks off of starch granules during crushing. The Samkwang cultivar had a higher damaged starch content than the other cultivars because Samkwang is a hard rice, while Shingil and Hangaru are soft rice. According to the study by Choi et al. [13], rice with low hardness has a lower amount of damaged starch. The particle size of Samkwang was the largest, $56.50 \mu \mathrm{m}$, while corn starch was significantly smaller, $39.28 \mu \mathrm{m}$. Particle size and damaged starch content of rice flour are known to affect water binding forces and gelatinization $[14,15]$. These differences in the raw rice flours depending on the amylose content, damaged starch content, and particle size of the different cultivar are believed to affect their quality properties of rice flour suspension according to heat or UHP treatment.

3.2. Microscopic Structure. Scanning electron micrographs of the samples from the different treatment methods are shown in Figure 1. In the untreated samples, the Samkwang cultivar has a polygonal starch shape and the Shingil and Hangaru cultivars have round shapes, like those of wheat starch [16, 17]. When heat or UHP $(600 \mathrm{MPa})$ treatments proceeded, it was confirmed that the structure of the starch collapsed and was irreversibly deformed. Choi et al. [18] showed that as starch becomes gelatinized, its structure unravels and collapses, such that its original structure cannot be observed. The deformations observed for the starch particles under pressure were similar to research trends presented by Li et al. [19] and Katopo et al. [20] for starch deformation under $600 \mathrm{MPa}$ treatment.

3.3. Moisture Absorption. Moisture absorptions for the rice flours under heat and UHP treatments are shown in Figure 2. Moisture absorptions for the heat treatment samples were the highest, occurring in the following order: corn starch $>$ Samkwang $>$ Hangaru $>$ Shingil, with values of 554.85, 519.45, 483.03, and 467.53\%, respectively. Moisture absorption rate measured the amount of water penetrated into interior or adsorbed on the surface of starch particles, and these abilities enhanced with increasing the amorphous part, 


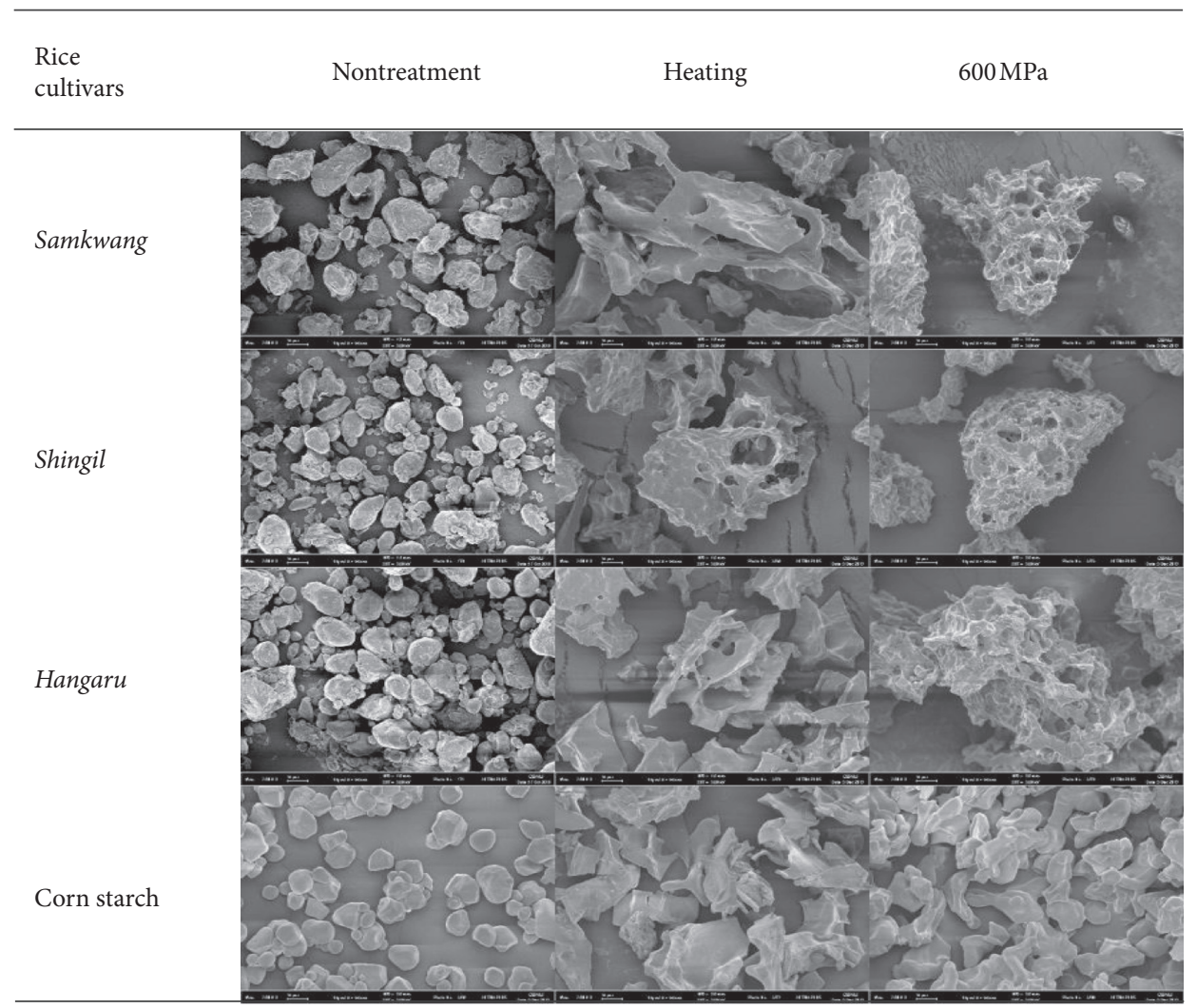

FIgURE 1: Scanning electron microscope of different rice flours by treatment methods $(\times 200)$.

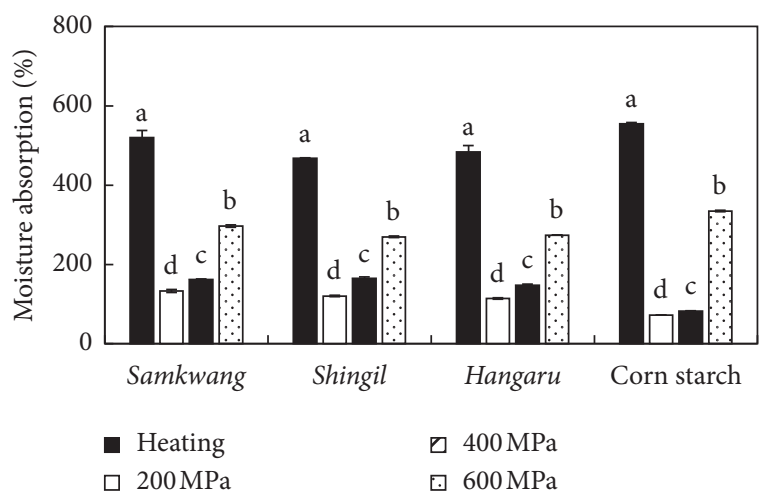

FIGURE 2: Moisture absorption of different rice flours by treatment methods. Values are mean \pm SD. $n=3$. Different small letters in the same items indicate a significant difference by Duncan's range test $(p<0.05)$ according to the treated method.

which is the branching part of amylopectin of the starch [5]. Therefore, Samkwang cultivar, which relatively has low amylose cultivar, was the highest value in moisture absorption rate. Overall, UHP treatment showed lower moisture absorption than heat treatment. The Samkwang cultivar had a significantly high value, $132.85 \%$, while corn starch had a low value, $72.27 \%$, under $200 \mathrm{MPa}$ treatment. As the applied pressure increased, moisture absorption increased. Corn starch had a significantly high value, $334.57 \%$, while Shingil had a low value, $269.55 \%$, under $600 \mathrm{MPa}$ treatment. Moisture absorption is expressed as the sum total of water absorbed to the amorphous portion of the particles, their surfaces, and interconnection between them. When starch is gelatinized via heating, water penetrates the starch infrastructure, forming hydrogen bonds with hydroxyl groups, causing the starch granules to expand [21]. Similarly, when pressure is applied to a starch suspension, water molecules penetrate the starch granules, form hydrogen bonds, and lead to particle expansion [6]. However, gelatinization via pressure has been reported to inhibit volume growth [22]. Therefore, moisture absorption is considered to be low.

3.4. Iodine Reaction. Results of the iodine reactions of the rice flours under heat and UHP treatments are shown in Figure 3. The absorbances of untreated samples were high, following the order: corn starch $>$ Shingil $>$ Hangaru $>$ Samkwang, with values of $0.648,0.357,0.500$, and 0.341 , respectively. Upon heat treatment, the absorbances of the samples decreased. Comparatively, UHP treatment samples showed an increase at $200 \mathrm{MPa}$, then decreased with increasing pressure. Shingil, which has a high amylose content, had the highest absorbance values. In the case of heat treatment, the absorbance values decreased due to dissolution of the chain structures of amylose. In the UHP treatment, as the pressure increased, the absorbance tended to decrease. The decreased absorbance with increased applied pressure was due to amylose leaching from swollen starch molecules $[23,24]$. Amylose binds to lipids to form an amylose-lipid complex, which seems to reduce iodine response [6]. 


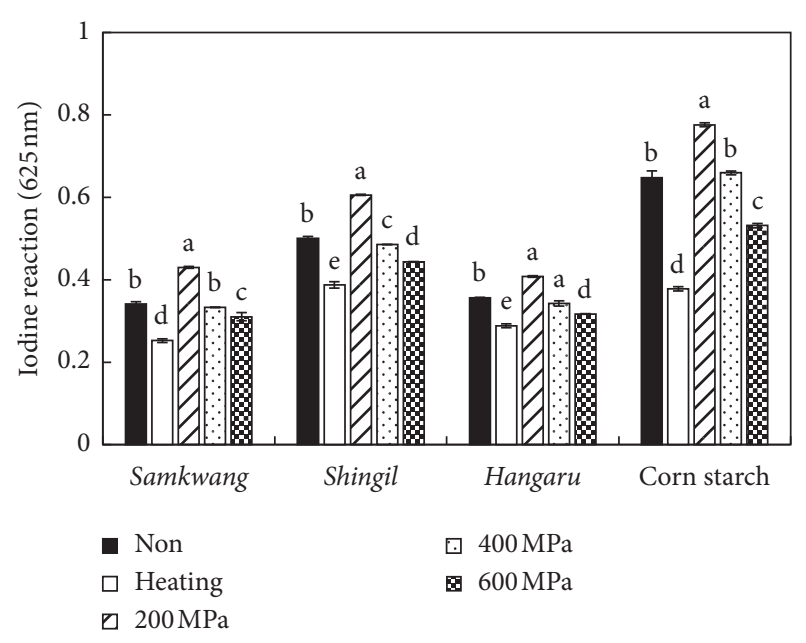

FIGURE 3: Iodine reaction $(625 \mathrm{~nm})$ of different rice flours by treatment methods. Values are mean \pm SD. $n=3$. Different small letters in the same items indicate a significant difference by Duncan's range test $(p<0.05)$ according to the treated method.

3.5. $\alpha$-Amylase Hydrolysis Rate. The $\alpha$-amylase hydrolysis rates of the rice flours under heat and UHP treatments are shown in Figure 4 . The $\alpha$-amylase hydrolysis rates of the untreated samples were $4.81-6.73 \%$. Under heat treatment, hydrolysis rates increased with starch gelatinization. Corn starch had the highest rate, $55.76 \%$, and new road cultivation had the lowest rate, $50.20 \%$. Gelatinized starch is easily digested by starch degrading enzymes due to the complete release of starch molecules [12]. According to Selmi et al. [25], enzymatic hydrolysis of corn starch shows a higher hydrolysis rate under heat treatment compared to $600 \mathrm{MPa}$ treatment. However, after a period of time, UHP treatment hydrolysis rates increase to heat treatment levels. Li et al. [26] also reported that hydrolysis of starch suspensions has a negative correlation to amylose content, which is thought to be due to increased enzymatic resistant through formation of amylose-lipid compounds [27].

3.6. Resistant Starch Content. The resistant starch contents of the rice flours under heat and UHP treatments are shown in Figure 5. Untreated samples had the highest resistant starch contents, following the order: corn starch $>$ Shingil $>$ Hangaru $>$ Samkwang, with values of 2.08, 0.87, 0.86, and $0.74 \%$, respectively. Heat treatment samples showed a significantly high value for corn starch, $3.19 \%$, and a low value for Hangaru cultivar, $2.53 \%$. However, there were no significant content differences among the rice cultivars. Among the UHP treated samples, the resistant starch contents increased with increasing pressure, with corn starch being the highest. Resistant starch content has a positive correlation to amylose content because degraded amylose consists of linear molecules of $\alpha$-1,4-d-glucan [28]. After UHP treatment, it is reported that starch deforms to a type-B structure, reducing its sensitivity to enzymes [29]. According to Li et al. [30], the resistant starch content of corn starch immediately increases after high hydrostatic pressure treatment, which is similar to the results observed in this experiment.

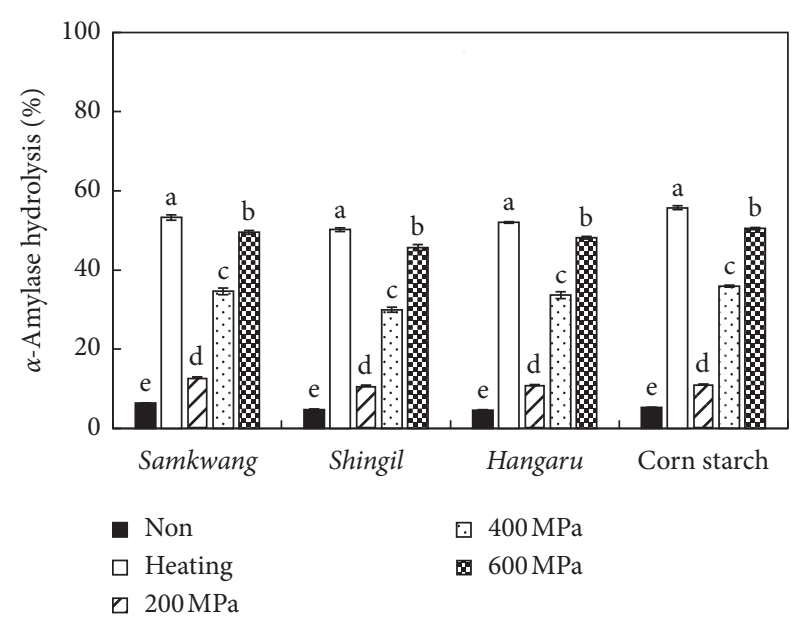

FIGURE 4: $\alpha$-Amylase hydrolysis of different rice flours by treatment methods. Values are mean \pm SD. $n=3$. Different small letters in the same items indicate a significant difference by Duncan's range test $(p<0.05)$ according to the treated method.

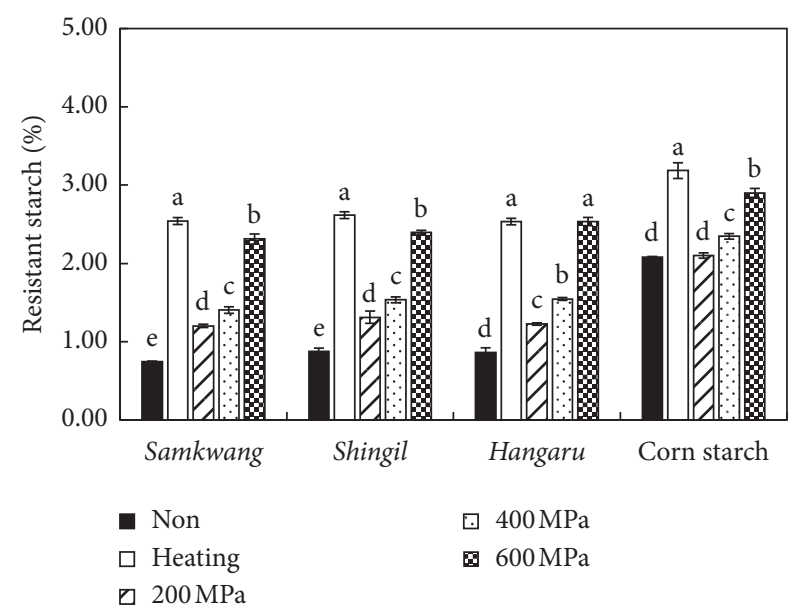

FIgURE 5: Resistant starch content of different rice flours by treatment methods. Values are mean \pm SD. $n=3$. Different small letters in the same items indicate a significant difference by Duncan's range test $(p<0.05)$ according to the treated method.

\section{Conclusion}

This study investigated the physicochemical properties of rice flour suspension according to cultivars and the foodprocessing method (heat and UPH treatment). Normal type (Samkwang cultivar) and processing type (Shingil and Hangaru cultivars) rice was shown to have different starch structures, and especially Shingil showed the highest amylose content $(22.98 \%)$ with round shape of microscopic structure like those of wheat starch. A comparison of heat and UHP treatments of rice suspensions showed the moisture absorptions of heat treatment samples (467.53-554.85\%) were higher than those of UHP treatment samples (72.27-334.57\%). As the applied pressure was increased, iodine reaction values decreased below those of the untreated samples and hydrolysis rates of $\alpha$-amylase increased to $45.69-50.48 \%$, which were lower than those of the heat 
treatment samples (50.20-55.76\%). The hydrolysis rate of $\alpha$-amylase was lowest for the Shingil cultivar, which contained a high amylose content, while little difference between the Samkwang and Hangaru cultivars was observed. Resistant starch contents of the UHP treatment samples were also similar to those of the heat treatment samples (2.53-3.19\%). Therefore, development of raw materials for new foods with different physicochemical properties is expected from rice cultivars via UHP processing.

\section{Data Availability}

No data were used to support the findings of this study.

\section{Conflicts of Interest}

The authors declare no conflicts of interest.

\section{Acknowledgments}

This work was carried out with the support of "Improvement of Utilization and Identification of Quality on Rice Flour and Products with Storage Periods (project no. PJ0128412020)" Rural Development Administration, Republic of Korea.

\section{References}

[1] R. Hayashi, "Utilization of pressure in addition to temperature in food science and technology," High Pressure Biotechnology, pp. 185-193, John Libbey Eurotext, London, UK, 1992.

[2] D. Knorr, "Effects of high-hydrostatic-pressure processes on food safety and quality," Food Technology, vol. 47, no. 6, pp. 156-161, 1993.

[3] G. W. Gould, "The evolution of high pressure processing of foods," in Ultra High Pressure Treatments of Foods, pp. 3-21, Springer, Berlin, Germany, 2001.

[4] S. K. Oh, "Development of rice varieties for processing and trend of food industry," Food Industry And Nutrition, vol. 21, no. 2, pp. 8-14, 2016.

[5] B.-H. Bang, K.-P. Kim, and E.-J. Jeong, "Quality characteristics of cookies that contain different amounts of chlorella powder," Korean Journal of Food Preservation, vol. 20, no. 6, pp. 798-804, 2013.

[6] H. E. Oh, Y. Hemar, S. G. Anema, M. Wong, and D. Neil Pinder, "Effect of high-pressure treatment on normal rice and waxy rice starch-in-water suspensions," Carbohydrate Polymers, vol. 73, no. 2, pp. 332-343, 2008.

[7] B. O. Juliano, "Criteria and tests for rice grain qualities," in Rice Chemistry and Technology, AACC, St Paul, MN, USA, 2nd edition, 1985.

[8] AACC International, Approved Methods of the American Association of Cereal Chemists, AACC International, St. Paul, MN, USA, 10th edition, 2011.

[9] D. G. Medcalf and K. A. Gilles, "Wheat starches I. Comparison of physicochemical properties," Cereal Chemistry, vol. 42, pp. 558-568, 1965.

[10] P. C. Williams, F. D. Kuzina, and I. Hlynka, "A rapid colorimetric procedure for estimating the amylose content of starches and flours," Cereal Chemistry, vol. 47, no. 4, pp. 411-421, 1970.

[11] Q. Xue, R. K. Newman, and C. W. Newman, "Effects of heat treatment of barley starches on in vitro digestibility and glucose responses in rats," Cereal Chemistry, vol. 73, no. 5, pp. 588-592, 1996.

[12] P. Guo, J. Yu, L. Copeland, S. Wang, and S. Wang, "Mechanisms of starch gelatinization during heating of wheat flour and its effect on in vitro starch digestibility," Food Hydrocolloids, vol. 82, pp. 370-378, 2018.

[13] O. J. Choi, H. N. Jung, and K. H. Shim, "Physicochemical properties of powdered, soft and hard type rice flour by different milling methods," Korean Journal of Food Preservation, vol. 22, no. 2, pp. 174-181, 2015.

[14] S. H. Lee and M. S. Shin, "Properties of preparation of rice manju and rice flour with soaking and different particle sizes," Korean Journal of Food and Cookery Science, vol. 25, no. 4, pp. 427-434, 2009.

[15] J. D. Park, B. K. Choi, J. S. Kum, and H. Y. Lee, "Physicochemical properties of brown rice flours produced under different drying and milling conditions," Korean Journal of Food Science and Technology, vol. 38, no. 4, pp. 495-500, 2006.

[16] H. A. Oh, M. Y. Kim, Y. J. Lee et al., "Quality characteristics of rice bread with different cultivars and milling methods," Korean Journal of Food Science and Technology, vol. 50, no. 5, pp. 492-498, 2018.

[17] Y.-J. Won, E.-K. Ahn, E.-G. Jeong et al., “An opaque endosperm rice cultivar, "Hangaru" suitable for exclusive drymilling rice flour production," Korean Journal of Breeding Science, vol. 51, no. 2, pp. 134-139, 2019.

[18] G. C. Choi, H. S. Na, G. S. Oh, S. K. Kim, and K. Kim, "Gelatinization properties of waxy black rice starch," Journal of the Korean Society of Food Science and Nutrition, vol. 34, no. 1, pp. 87-92, 2005.

[19] J. H. Li, T. Vasanthan, R. Hoover, and B. G. Rossnagel, "Starch from hull-less barley: V. In-vitro susceptibility of waxy, normal, and high-amylose starches towards hydrolysis by alpha-amylases and amyloglucosidase," Food Chemistry, vol. 84, no. 4, pp. 621-632, 2004.

[20] H. Katopo, Y. Song, and J. L. Jane, "Effect and mechanism of ultrahigh hydrostatic pressure on the structure and properties of starches," Carbohydrate Polymers, vol. 47, no. 3, pp. 233-244, 2002.

[21] W. S. Ratnayake, R. Hoover, and T. Warkentin, "Pea starch: composition, structure and properties-a review," Starch-Stärke, vol. 54, no. 6, pp. 217-234, 2002.

[22] F.-J. Tan, W.-T. Dai, and K.-C. Hsu, "Changes in gelatinization and rheological characteristics of japonica rice starch induced by pressure/heat combinations," Journal of Cereal Science, vol. 49, no. 2, pp. 285-289, 2009.

[23] J. P. Douzals, P. A. Marechal, J. C. Coquille, and P. Gervais, "Microscopic study of starch gelatinization under high hydrostatic pressure," Journal of Agricultural and Food Chemistry, vol. 44, no. 6, pp. 1403-1408, 1996.

[24] B. A. Bauer, M. Hartmann, K. Sommer, and D. Knorr, "Optical in situ analysis of starch granules under high pressure with a high pressure cell," Innovative Food Science \& Emerging Technologies, vol. 5, no. 3, pp. 293-298, 2004.

[25] B. Selmi, D. Marion, J. M. Perrier Cornet, J. P. Douzals, and P. Gervais, "Amyloglucosidase hydrolysis of high-pressure and thermally gelatinized corn and wheat starches," Journal of Agricultural and Food Chemistry, vol. 48, no. 7, pp. 26292633, 2000.

[26] W. Li, Y. Bai, S. A. S. Mousaa, Q. Zhang, and Q. Shen, "Effect of high hydrostatic pressure on physicochemical and structural properties of rice starch," Food and Bioprocess Technology, vol. 5, no. 6, pp. 2233-2241, 2012. 
[27] R. Cui and C. G. Oates, "The effect of amylose-lipid complex formation on enzyme susceptibility of sago starch," Food Chemistry, vol. 65, no. 4, pp. 417-425, 1999.

[28] P. Raigond, R. Ezekiel, and B. Raigond, "Resistant starch in food: a review," Journal of the Science of Food and Agriculture, vol. 95, no. 10, pp. 1968-1978, 2015.

[29] W. Błaszczak, S. Valverde, and J. Fornal, "Effect of high pressure on the structure of potato starch," Carbohydrate Polymers, vol. 59, no. 3, pp. 377-383, 2005.

[30] W. Li, X. Tian, P. Wang et al., "Recrystallization characteristics of high hydrostatic pressure gelatinized normal and waxy corn starch," International Journal of Biological Macromolecules, vol. 83, pp. 171-177, 2016. 